\title{
The Circumplex Structure of Depersonalization/Derealization
}

\author{
Robert G. Sacco \\ School of Behavioral and Health Sciences, Northcentral University \\ 10000 E. University Drive, Prescott Valley, Arizona 86314, USA \\ E-mail: robertgsacco@gmail.com
}

\begin{abstract}
This paper presents a literature review to determine whether precipitating causes of depersonalization (DP) and derealization (DR) correspond to the circumplex model of emotion defined by the dimensions of valence and arousal. DP/DR precipitators are reviewed and classified in relation to the four quadrants of the circumplex model of emotion. The findings indicate that the valence and arousal dimensions were significant predictors of emotions expressed in DP/DR precipitators. Implications are discussed with respect to the multidimensional nature of DP/DR, clarifying normal and abnormal DP/DR, and identifying needed areas of research.
\end{abstract}

Keywords: Affect, Arousal, Circumplex model of emotion, Depersonalization, Derealization

\section{Introduction}

Depersonalization/derealization disorder (DP/DR-D) involves persistent episodes of depersonalization (DP) and/or derealization (DR), states that represent a changed overall experience in self-awareness. DP includes an altered, estranged, or detached subjective experience of one's self, one's mental processes, and one's body during which people feel that they are robots or that they are in a dream. DR often accompanies DP in which the external world also appears strange or unreal (American Psychiatric Association [APA], 1994; World Health Organization [WHO], 1992). People with DP/DR-D typically report feeling as if they were spectators observing a play of the self or as if they were observing themselves from a distance. They may fear a loss of control over their thoughts and actions or that they are going crazy. Their capacity for reality testing, however, remains intact (e.g., knowing that one is not literally a robot). As well as these core diagnostic criteria, sufferers often report marked distress or impairment in social, occupational, or other functioning. Studies reveal the prevalence of DP/DR-D is within the range of 1-2\% (Hunter, Sierra, \& David, 2004).

Mild and transient symptoms of DP/DR are common in the general population, with an annual prevalence of 45-75\% (Hunter et al., 2004). The symptoms of DP/DR occur across a wide range of causes such as hypnosis (Wineburg \& Straker, 1973), meditation (Castillo, 1990), hypnagogic and hypnopompic states (Lambert, Senior, Phillips, \& David, 2000), sleep deprivation (Bliss, Clark, \& West, 1959), sensory deprivation (Reed \& Sedman, 1964), hyperventilation (Lickel, Nelson, Lickel, \& Deacon, 2008), stress (Aderibigbe, Bloch, \& Walker, 2001), and drug or alcohol use (Melges et al., 1974). The relatively high rates of reporting of these symptoms, in combination with the diverse range of precipitants, increase the need for clarification between normal and pathological episodes of DP/DR. In other words, why are the normally transient symptoms of DP/DR perpetuated in some people but not in others?

The cognitive-behavioral conceptualization of DP/DR-D (Hunter, Phillips, Chalder, Sierra, \& David, 2003) posits the symptoms are perpetuated because of misinterpretations of the normally transient symptoms of DP/DR. According to this view, most people experience temporary DP/DR but most people attribute it to situational stressors and pay little attention to it, thus leading to a decrease in symptoms as the situational factors alleviate. On the other hand, people who develop the chronic condition are thought to catastrophically misinterpret the transient symptoms of DP/DR, for example, taking them as indicative of serious mental illness and/or brain dysfunction. This thinking is suggested to lead to anxiety, which in turn fuels continuation and worsening of symptoms.

This account, however, appears to oversimplify the nature of pathological DP/DR. The cognitive-behavioral model (Hunter et al., 2003) rests on the premise that pathological DP/DR is only a variation (i.e., cognitive misinterpretation) of the same underlying symptoms as those experienced in transient episodes: "The symptoms of depersonalisation disorder are the same as those experienced in transient episodes, except that in DPD the symptoms are experienced chronically, cause significant distress and result in functional impairment" (p. 1453). The problem is this account overlooks the phenomenological complexity of the symptoms. In particular, it ignores the possibility that while patients may describe the same "symptoms" as those experienced in transient 
episodes (e.g., feelings of unreality), the "quality" of the symptoms may differ (Steinberg, 1995). Thus, the relationship between pathological and transient symptoms of DP/DR appears to require further explanation.

Steinberg (1995) proposed the symptoms of pathological DP/DR involve distinguishing qualitative features. Supporting this view is the fact that not all people experience DP/DR as unpleasant. Strictly speaking, DP/DR is noted to have in particular an unpleasant quality (Ackner, 1954). Yet while the symptoms of DP/DR are regarded as being unpleasant slightly more than half of the people reporting DP/DR experiences actually rate them as being pleasant and not unpleasant (Trueman, 1984). Qualitatively, there is thus a discrepancy between some types of DP/DR experiences, suggesting distinct underlying mechanisms. Evidence like this led Steinberg (1995) to suggest that precipitants of "common-mild" DP/DR, usually caused by fatigue, sleep deprivation, meditation, sensory deprivation, stress, and drug use could not account for pathological DP/DR, because of qualitatively distinct features and causes in the latter. Indeed, although stress or drug use may precipitate the condition, the condition can become chronic with no identifiable stress or history of drug use (Simeon \& Abugel, 2006).

One way to clarify distinctions between normal and pathological DP/DR is to consider the manner in which DP/DR precipitants correspond to the valence and arousal dimensions of affect. Emotional affect is conceptualized along two dimensions: 1) valence, which describes the extent of pleasure or displeasure, and 2) arousal, which describes the level of arousal (Russell, 1980; Russell \& Barrett, 1999). These two dimensions, theorized to be bipolar and orthogonal, take the form of a "circumplex": a two-dimensional geometric structure in the form of a circle. All human emotional experience (i.e., emotions such as anger, sadness, and fear, as well as nonemotional affective states like fatigue, sleepiness, and relaxation) can be defined as combinations of these two independent dimensions. The valence/arousal circumplex model has a long history in affective psychology and researchers advocate use of the circumplex model particularly for a systematic arrangement of conscious emotional experience (for review, see Russell \& Barett, 1999).

The circumplex model is not the only way to understand emotion. The emotion circumplex provides a theoretical description of affect from a structural standpoint, but it does not provide a description of the function of affect (Carver, 2001). Nevertheless, understanding affect in structural terms is considered to be important for viewing emotion concepts because it clarifies relationships between various affects. No model has yet emerged that classifies the different causes of DP/DR (or, for that matter, "altered states of consciousness") in relation to the emotion circumplex. Fischer (1971) outlined a useful map of altered states of consciousness, under which alterations occur along the dimensions of either hyper or hypoarousal. There was however no valence dimension to the model. More recently, Vaitl et al. (2005) provided an extensive review of a wide range of phenomena included under the rubric of altered states of consciousness. The aim of that paper, however, was to describe the neurophysiological, cognitive, biological, as well as phenomenological underpinnings of altered states of consciousness in the empirical literature rather than classifying the phenomena from a structural standpoint.

The purpose of the present article is to review the research literature for evidence that precipitating causes of DP/DR could be fitted to a circumplex structure consisting of valence and arousal dimensions. Since there is an extensive catalog of precipitators reported to cause DP and/or DR, and little or no theoretical basis for bringing them under scrutiny, the taxonomy of a circumplex will not only offer a way of conceptually classifying the various precipitators, but in so doing, will also help to clarify distinctions between normal and pathological DP/DR.

\section{Circumplex-Derived Dimensions}

To test the hypothesis that the emotion circumplex structure generalizes to DP/DR, a comprehensive review of the research literature was conducted. The literature review involved electronic database searches (e.g., PubMed, PsycINFO, Web of Science, Google Scholar) for peer-reviewed articles that included depersonalization or derealization in the key words, title, or abstract. Additional articles were identified in the reference lists of relevant publications. Electronically searchable books were also included. Over 200 journal articles and a number of books and book chapters were found. The titles and abstracts of all these references were screened for relevance and imported electronically into a bibliographic database (Mendeley, Version 0.9). Full publications were then retrieved for those which appeared to address the purposes of this review.

Based on the literature review, 19 DP/DR precipitators were identified that have received consistent support in reviews on the topic but have not been previously linked to the emotion circumplex. Figure 1 shows the 19 precipitators in the two-dimensional space. As predicted, the 19 DP/DR precipitators fit the circumplex structure. The vertical axis represents the arousal dimension ranging from high (top) to low (bottom). At the high end of the vertical arousal dimension, DP/DR precipitators such as "sports" and "overwhelming joy" can be seen. Precipitators such as "fatigue" and "hypnagogic/hypnopompic states" can be seen at the low end. The horizontal 
dimension represents the valence property of the precipitators ranging from negative (left) to positive (right). Negative precipitators are items such as "stress" and "heightened self-focus," whereas positive precipitators are items such as "overwhelming joy" and "hypnotic induction."

Figure 1 shows the correlation between the 19 DP/DR precipitators. Precipitators that fall close together are likely to be expressed at the same time. In contrast, precipitators that fall further apart are less likely to be expressed at the same time. For example, the precipitators "extreme threat" and "hyperventilation" are close together on the negative side of the circumplex. Thus, "extreme threat" is much more likely to accompany "hyperventilation" than by "overwhelming joy" (which is located on the opposite side of the circumplex).

The preceding discussion has articulated some general hypotheses about the circular ordering of DP/DR in two-dimensional space. The next task is to evaluate evidence for the validity of the circumplex-derived valence and arousal dimensions. This is discussed in the sections that follow by consulting several empirical literatures.

\subsection{Pleasant-Aroused Quadrant}

In Figure 1, the circumplex is divided into four quadrants, with each representing different combinations of valence and arousal. This first section examines evidence relating to four DP/DR precipitators located in the pleasant-aroused quadrant: overwhelming joy, sports, positive drug experiences, and dancing.

\subsubsection{Overwhelming Joy}

At first glance it seems paradoxical to suggest that DP/DR could result from positive experiences. After all, most theorists have assumed that dissociative experiences are defense mechanisms elicited by cues of threat (Van IJzendoorn \& Schuengel, 1996). Dissociation during positive events, however, has been found to occur (Pica \& Beere, 1995). According to Beere's (1995a, 1995b; Pica \& Beere, 1995) perceptual theory of dissociation, an event does not need to be subjectively traumatic or threatening to invoke dissociative characteristics. Rather, it is the overwhelming or "captivating" nature of the stimulus that triggers dissociative reactions. In such circumstances perception becomes "captivated by a particular aspect of the lived situation" and consequently, "different components of the perceptual background become lost, resulting in the dissociative reaction" (Beere, 1995b, p. 243). By surveying undergraduates about positive dissociative experiences, Pica and Beere (1995) showed that this is not only possible, but relatively common. In fact, their study reported that $37 \%$ of the participants endorsed positive dissociative experiences, most commonly in experiences involving sports, sex, prayer, nature, positive news, acting, hobbies, and music.

\subsubsection{Sports}

Altered states of consciousness resembling DP/DR experiences are common in sports. Using interview techniques, Ravizza (1977) researched the personal experiences of athletes involved in various sports such as golf, swimming, track and field, jogging, surfing, and skiing. Several of the athletes' descriptions of their experiences revealed similarities to DP/DR. They reported experiencing time and space disorientation, Godlike feelings, ego loss, and feeling at one with the universe. Intriguing evidence of dissociation in sporting events was provided by Sterlini and Bryant (2002) in a study on dissociation in novice sky divers. In their study, they found that during their first skydive $38 \%$ of the participants experienced time distortions and $37 \%$ of the participants experienced derealization. As with cases of overwhelming joy, sports related alterations in consciousness appear to result from the narrowing of perception that isolates background components.

\subsubsection{Positive Drug Experiences}

Use of alcohol and other drugs is often motivated by the desire to produce positive or pleasurable moods. Many drugs, besides inducing an emotional state of well-being or euphoria, can alter the sense of self and induce depersonalization and/or derealization (Mathew et al., 1999; Vollenweider, Vontobel, Hell, \& Leenders, 1999). Drug-induced depersonalization is sometimes, although not always, a pleasant sensation (Mathew et al., 1999). One reason people might find alterations in the sense of self following drug use subjectively pleasant is a reduction of self-awareness. Often, the state of self-awareness is aversive, and thus, people may seek to escape or minimize it (Duval \& Wicklund, 1972). According to the self-awareness reduction model (Hull, 1987) part of the appeal of consuming alcohol or drugs is that it reduces self-awareness, thereby enabling people to forget their troubles. Baumeister (1991) has further observed that certain forms of bliss are facilitated by a loss of self-awareness, and so a loss of self-awareness may be an intrinsically pleasant, desirable state.

\subsubsection{Dancing}

Humans have practiced music and dancing for centuries to alter states of consciousness. Vaitl et al. (2005) describe dancing as a "rhythm-induced trance." In dancing, the body movements of the dancer rhythmically 
synchronize to the beat of the music and can "seem to happen automatically, without effort or voluntary control" (Vaitl et al., 2005, p. 107). Further, "self-reflective thinking ceases when the subject becomes increasingly absorbed in the action" and subjective changes can include "a distortion of the time sense, unusual bodily sensations (e.g., feeling light, warm, energized), vivid imagery, and strong positive emotions (e.g., joy, happiness, ecstasy) in conjunction with the impression of becoming one with the rhythm" (Vaitl et al., 2005, p. 107). Trance music is a genre of electronic dance music characterized by strong repetitive rhythmic percussion and is produced for the specific purpose of encouraging positive dissociative trance-like states among dancers at raves (Becker-Blease, 2004). Some people at raves and dance clubs also use psychoactive drugs like MDMA (ecstasy) because they further promote the trance-like states that dancers are seeking (Becker-Blease, 2004).

\subsection{Pleasant-Unaroused Quadrant}

This section provides evidence on three DP/DR precipitators located in the pleasant-unaroused quadrant: meditation, sensory deprivation, and hypnotic induction.

\subsubsection{Meditation}

Meditation is considered a psychologically induced altered state of consciousness (Vaitl et al., 2005). There is a wide variety of meditation techniques, and all involve voluntary changes in the quality and contents of consciousness. The subjective experience during transcendental meditation involves a change from the ordinary in a person's mental focus by cultivating a low arousal state, so that metabolic activity is slowed and self-awareness is reduced. The physiological research on its effects characterized transcendental meditation as being a "wakeful hypometabolic state" (Wallace, 1970). There is general agreement the meditative trance is associated with "blissful mental quiescence" (Vaitl et al., 2005, p. 108). Most meditation practioners look forward to the depersonalization experience and find it pleasurable (Castillo, 1990).

\subsubsection{Sensory Deprivation}

When faced with sensory deprivation, people sometimes experience depersonalization (Reed \& Sedman, 1964). Sensory deprivation refers to any major decrease in the amount or intensity of sensory stimulation. Intense or prolonged sensory deprivation is aversive and disorienting. Yet brief periods of restricted sensation may be pleasantly calming (Vaitl et al., 2005). Like meditation, people who undergo mild sensory deprivation often look forward to the depersonalization and associated sense of peace.

\subsubsection{Hypnotic Induction}

Often hypnotic induction will induce transient depersonalization symptoms (Wineburg \& Straker, 1973). This has been considered a useful exercise because by inducing the symptoms, the therapist can provide patients with a context for understanding and controlling them. People for whom this approach is effective are taught to deflect depersonalization symptoms by replacing them with pleasant imagery (Spiegel \& Spiegel, 2004). Other relaxation techniques (e.g., biofeedback) may also be helpful.

\subsection{Unpleasant-Unaroused Quadrant}

This section provides evidence on four DP/DR precipitators located in the unpleasant-unaroused quadrant: hypnagogic and hypnopompic states, fatigue, sleep deprivation, and heightened self-focus.

\subsubsection{Hypnagogic and Hypnopompic states}

People who have unusual sleep-related experiences in general score high on measures of dissociation (of which DP and DR are symptom clusters) (Watson, 2001; Giesbrecht \& Merckelbach, 2004). Hypnagogic and hypnopompic states refer, respectively, to the states just before falling asleep and just before waking up. These states are said to differ from dreams in that hypnagogic and hypnopompic experiences are usually static and lacking in narrative content (Vaitl et al., 2005). Imagery occurring in the hypnopompic state, however, may be difficult to separate from continuation of dream content. One study found that among 22 people with DP/DR-D, 5 reported hypnagogic or hypnopompic visual hallucinations (Lambert et al., 2000). Most people report some mild discomfort associated with this DP/DR.

\subsubsection{Fatigue}

Fatigue induced by prolonged or demanding activity may lead to depersonalization. Depersonalization is among the components of the burnout syndrome (Maslach \& Jackson, 1981; Maslach, 2003). Burnout is an outcome of overwhelming stress or work that results in the gradual depletion of a person's physical, emotional, and energetic resources. Theory suggests that people experience subjective fatigue as a general response to stress over time (Cameron, 1973). Subjective fatigue is experienced as decreased energy, wakefulness, alertness, and vigilance. 


\subsubsection{Sleep Deprivation}

Sleep deprivation is another cause of DP/DR (Bliss et al., 1959). Most sleep deprivation subjects experience a general rise in irritability and cognitive impairment. More dramatic effects tend to occur with prolonged and severe sleep deprivation, such as disorientation, perceptual hallucinations, paranoia, and distortions in perception of time and self (Oswald, 1970). Sleep deprivation produces marked impairments of "temporal memory" (memory for when events occur) (Harrison \& Horne, 2000). In general, the dissociation in memory for temporal order could produce strange and unfamiliar feelings about the self by impairing the capacity to represent the self as continuing through time.

Sleep deprivation normally lowers physiological arousal, although sometimes it may increase anxiety and tension. In a recent neuroimaging study, for example, subjects who were deprived of sleep for 35 hours showed a $60 \%$ increase in amygdala reactivity to aversive stimuli compared to controls (Yoo, Gujar, Hu, Jolesz, \& Walker, 2007). The increased activity in the amygdala, which plays a central role in regulating emotional brain function, provides evidence that sleep loss may strongly influence a person's emotional stability. It follows that people low on emotional stability will likely be more anxious and express more negative affectivity. Figure 1 highlights this point with a higher location of sleep deprivation relative to fatigue in the negative/low-arousal quadrant.

\subsubsection{Heightened Self-Focus}

An essential feature of DP/DR is heightened self-focus. This increased self-focus is regarded as an expression of obsessional self-monitoring (Torch, 1978, 1981). Patients often describe it as a split of their subjective awareness into two parts: an "observing self" and an "experiencing self." This split between an observing and experiencing self has more recently been dubbed "hyperreflexivity," which is an excessive tendency to take the self as an object of awareness (Sass, 1994). Due to a continual self-observation and compulsive self-analysis, the normal integrated whole of our experiences can be split apart. The resulting predicament involves a reduction of practical connectedness to the world, an alienation from one's own body, and solipsistic tendencies. As Parnass and Sass (2001, p. 105) put it, the person's perception is "not lived but is more like a mechanical, purely receptive sensory process, unaccompanied by its affective feeling-tone." This altered experiential realm is the backdrop against which DP and DR is cultured.

Deserving particular attention is whether self-focus is more a symptom or cause of DP/DR. Many of the findings in the research literature reviewed here make reference to self-focus as a symptom of DP/DR-D. The psychiatrist Evan Torch, however, offered a counterpoint to theorists who considered self-focus to be an isolated symptom of the disorder. Torch (1978) argued it is more accurate to view self-focus as a cause of DP/DR: "In depersonalization, self-observation is listed as a concurrent or resultant symptom. It is usually, rather, a primary causative factor" (p. 197). More work needs to be done on this wider understanding of self-focus as a psychological cause of DP/DR. But for now it does appear reasonable to suggest the influence of DP/DR is most acute and most persistent when people are specifically engaged in self-focus.

In general, self-focus is related to both negative affect and increased arousal (Ingram, 1990; Wells \& Matthews, 1994). Given that self-focus is related to increased arousal, it may seem misleading to place "self-focus" in the negative/low-arousal quadrant. In keeping with their subjective complaints of emotional numbing, however, chronic DP/DR sufferers may have reduced autonomic responses to stimuli that normally increase arousal (Sierra et al., 2002). Hence the resulting low-arousal placement of self-focus on the circumplex.

\subsection{Unpleasant-Aroused Quadrant}

This section reviews a final quadrant of the circumplex. This quadrant consists of eight DP/DR precipitators located in the unpleasant-aroused quadrant: stress, altered perceived causality, traumatic memory, extreme threat, hyperventilation, vertigo, epilepsy, and negative drug experiences.

\subsubsection{Stress}

Stressful life events or continuing stressors in one's life is one of the most commonly cited triggers of DP/DR (Aderibigbe et al., 2001). Although it has long been known that stress has some kind of link to DP/DR, the nature of this link has been far from clear. Models of stress (Lazarus, 1974) associate stress with affective states of increased arousal, often experienced as anxiety. One possible mechanism by which stress can cause DP/DR involves the causal process by which stress induces self-focus. Psychological stress regularly results in heightened self-focus. In stressful environments, self-focus directs attention toward discrepancy between preferred and actual self-standards (Carver \& Scheier, 1998). As discussed above (see section 2.3.4), heightened self-focus is a major source of DP/DR. 


\subsubsection{Altered Perceived Causality}

There is some similarity between the phenomenology of DP/DR and schizotypal personality disorder or schizophrenia (Simeon \& Hamilton, 2008). It would seem that magical thinking (i.e., belief in forms of causation considered invalid by conventional science) may account for the phenomenological similarity between DP/DR and schizotypy. Content measures developed to assess magical thinking in schizotypy have long recognized the association between magical thinking and unreality experiences (Gruzelier \& Doig, 1996).

Other types of altered perceived causality or "anomalous experience" include déjà vu and mystical experience. The experience of déjà vu has been reported by several authors as being accompanied by DP/DR (Brown, 2004). Mystical experience is also marked by a temporary experience of self-loss or ego-breakdown involving a loss of boundaries and altered sense of time (Hood, 1976, p. 182-183). There is evidence the effects of anomalous experience can include distress, agitation, and fear (Kennedy \& Kanthamani, 1995). Targ, Schlitz, and Irwin (2000) state: "Clinical observations of reactions to these experiences include fears of being hurt or going crazy, feelings of loss of control, disorientation from one's previous understanding of the world, and bewilderment" ( $\mathrm{p}$. 226).

\subsubsection{Traumatic Memory}

Traumatic memory may precipitate DP/DR (Steinberg, 1995). In traumatic memory a person may experience the intrusions of dissociated memories of the trauma, occurring as flashbacks, into consciousness. Flashbacks involve "varying degrees of a dreamlike state with immersion in past experience that effectively dissociates the self from present reality" (Simeon, 2007, p. 80). Traumatic memories occur within a broader context of stress, intense fear, and physiological hyperarousal cued by the traumatic memory.

\subsubsection{Extreme Threat}

Fleeting experiences of DP/DR are commonplace among people facing extreme threat (Noyes \& Kletti, 1977). There are two explanations to explain the high rates of DP/DR during extreme threat. In the first, the "protective response theory" of dissociation (Sierra \& Berrios, 1998; Sierra, 2009), DP/DR represents a preformed response of the brain designed to defend against threat. According to this view, DP/DR results from a "hard-wired" biological defense mechanism which evolved to minimize the potentially debilitating effects of extreme anxiety in threatening situations. A second view, the "perceptual theory" of dissociation (Beere, 1995a, 1995b), suggests that DP/DR can occur because during extreme threat, perception fixes to the startling figure. In this situation, even though perception focuses on the world, the world's background, according to this theory, is lost since perception focuses narrowly on the threat. According to this view, although intense DP/DR can occur during extreme threat it is a nonspecific dissociative state with no protective function.

The idea that extreme threat or trauma is related to DP/DR-D is prominently present in the literature. Sierra and Berrios (1998) maintain that DP/DR is a biological defense mechanism resulting from an evolution-prepared, threat-related dissociation. As Noyes and Kletti (1977) correctly pointed out, however, threat-related dissociation: "develops instantly upon recognition of danger and vanishes just as quickly when the threat to life is past" (p. 381-382). DP/DR-D, in contrast, does not vanish quickly; it persists without immediate threat. Also, DP/DR-D has not been compellingly linked to trauma. Emotional abuse is the only form of trauma found to be significantly related to DP/DR-D (Simeon, Guralnik, Schmeidler, Sirof, \& Knutelska, 2001). Recognizing that emotional deprivation can have a causal role in emotional, social, and personality development that may significantly impact the course of induction of DP/DR-D, this causal role is still at odds with the assumed defensive function of dissociation. More recently the assumed defensive function of dissociation has been criticized (Giesbrecht, Lynn, Lilienfeld, \& Merckelbach, 2010).

\subsubsection{Hyperventilation}

Hyperventilation can also cause a sense of depersonalization and derealization (Lickel et al., 2009). When high anxiety-sensitive undergraduate students were required to voluntarily hyperventilate the hyperventilation was found to be effective in inducing depersonalization and derealization (Lickel et al., 2009). Hyperventilation is an incorrect way of breathing either too rapid or too deep. When you breathe faster or deeper than necessary your body will inhale too much oxygen and this will cause the carbon dioxide levels in the body to drop. As a response the body will narrow the blood vessels which will cause symptoms such as headache, dizziness, confusion, and increased heart rate. Involuntary hyperventilation is a normal response when we are anxious. It is a protective device signaling a threat and preparing the body to act if needed. Hyperventilation is among the most common symptoms observed during panic attacks, as are depersonalization and derealization (Barlow, 1988). 


\subsubsection{Vertigo}

Vertigo is a type of dizziness involving a sensation that one's surroundings are moving or spinning while one is in fact stationary. Vertigo is due to a dysfunction of the vestibular system in the inner ear (Neuhauser \& Lempert, 2009). The most common causes of vertigo linked to DP/DR are vestibular dysfunction (Grigsby \& Johnston, 1989) and head trauma (Grigsby \& Kaye, 1993). Not surprisingly, it has been suggested the pathophysiology explaining DP/DR after head trauma is vestibular symptoms, such as vertigo and dizziness (Hoffer, Balough, \& Gottshall, 2007). Vertigo as related to DP/DR is reflective of the more general relationship between DP/DR and dizziness (Fewtrell \& O'Connor, 1988).

Most people report the sudden onset of vertigo as highly disturbing and report associated nausea and vomiting as well as difficulties standing or walking. In addition, an affective or anxiety-related component of vertigo has long been recognized (Balaban \& Jacob, 2001).

\subsubsection{Epilepsy}

DP and DR is reported to occur with temporal lobe epilepsy (TLE) and seizures (Devinsky, Putnam, Grafman, Bromfield, \& Theodore, 1989; Kenna \& Sedman, 1965). The role of the temporal lobe in DP was described by the neurologist Wilder G. Penfield and his colleagues at the Montreal Neurological Institute. During surgical treatment for epilepsy, Penfield and Rasmussen (1950) noted that direct electrical stimulation of various areas of temporal lobes produced phenomena resembling DP such as "illusions of unfamiliarity, strangeness, and remoteness" (p. 173). Accordingly, these insights were formulated as the "temporal lobe hypothesis" of DP.

The emotions aroused by epileptic seizures themselves are most often fear (Williams, 1956). One study found that DP (but not DR) occurred in 11 of 30 patients with TLE and that the DP was usually brief and associated with fear (Harper \& Roth, 1962).

\subsubsection{Negative Drug Experiences}

Drug use can cause a potentially negative feeling of DP/DR, particularly after large doses or during the first few exposures to the drug. The most common psychoactive drugs to cause DP/DR are cannabis (Melges, Tinklenberg, Hollister, \& Gillespie, 1970b) and hallucinogens (Vollenweider et al., 1999), but DP/DR episodes can also be precipitated by ketamine ("special K") and MDMA ("ecstasy") (Medford et al., 2003). Empirical studies clearly document that cannabis heightens DP/DR (Mathew et al., 1999). For some people, DP/DR induced by cannabis correlated with anger, tension, and confusion, suggestive of negative components (Mathew et al., 1999). Melges and colleagues (1970a) were the first to confirm a link between THC and "temporal disintegration," or a disorganization of sequential thought and impaired goal-directedness. This phenomenon stems partly from impaired immediate memory. Melges and colleagues (1970b) also showed that DP/DR is closely associated with the level of temporal disintegration. Thus it appears that temporal disintegration is fundamentally involved in the precipitation of DP/DR.

A major question is whether negative drug experiences can lead to chronic DP/DR. Medford et al. (2003) identified 40 cases where subjects attributed the onset of their DP/DR-D to illicit drug use. Thus, the subjective response reported by these participants was the onset of chronic DP/DR symptoms followed illicit drug use. Still, the causal process underlying involuntary perpetuation of the symptoms remains to be elaborated. An intriguing line of research (reviewed by Wells \& Matthews, 1994, p. 225) suggests that drug use (e.g., marijuana) can influence attentional processes. Research shows that marijuana intoxication increases "absorption," a state in which a person's perceptual and ideational resources are totally engaged. The issue, presumably, is that some marijuana users may be more inclined toward intense self-focus or "self-absorption" (i.e., an excessive, continued, and rigid focus on the self) depending on whether their object of absorption is self or non-self-relevant. Research has shown that introspective marijuana users are more likely than less introspective users to develop mental health problems because of the greater inclination for intense self-focus and self-evaluation (Zablocki, Aidala, Hansell, \& White, 1991). These findings suggest the inclination for self-focus may play an important role in the process through which marijuana induced changes in the self translate into temporary psychological distress and, if experienced repeatedly, into chronic psychological symptoms.

\subsection{Summary}

In summary, the literature review and inspection of the two-dimensional space in Figure 1 provide evidence that the valence and arousal dimensions represent two basic and fundamental qualities on which the $19 \mathrm{DP} / \mathrm{DR}$ precipitators vary. The organization of the $19 \mathrm{DP} / \mathrm{DR}$ precipitators appear properly placed and are well supported by the four quadrants of the circumplex model of emotion. 


\section{Implications of the Model}

The present taxonomy serves several useful functions. Among these are its ability: 1) to capture the multidimensional nature of DP/DR, 2) to clarify normal and abnormal DP/DR, and 3) to identify needed areas of research and conceptual development.

\subsection{The Multidimensional Nature of Depersonalization/Derealization}

First and foremost, the present investigation supports a multidimensional conception of DP/DR. Most theories of DP/DR have proposed that these symptoms are unidimensional in nature, in the sense that different factors that affect DP/DR have their effects on the same underlying psychological (e.g., Hunter et al., 2003) or biological (e.g., Sierra \& Berrios, 1998) processes. The present research, however, contradicts such views. Instead, it appears that DP/DR are extremely varied and are produced by several causal processes (e.g., psychological and biological). There is substantial evidence for the usefulness of treating DP/DR as multidimensional in nature because many phenomenologically similar symptoms of DP/DR are actually quite different from each other (since they arise from different mechanisms - epilepsy, drug use, meditation, vertigo, major life stress, self-focus, etc.). Specification among various causes of DP/DR is important because, as the review results indicate, the affective implications of DP/DR depend not only on the subjective intensity of DP/DR, but also, and maybe more importantly, on the cause of DP/DR. When people depersonalize during overwhelming joy, for example, they experience intense positive affect; whereas when people depersonalize during extreme threat, they experience intense negative affect. Thus, depersonalization has different affective implications depending on the context.

The circumplex model of DP/DR improves on existing classification efforts (Sierra, Baker, Medford, \& David, 2005; Simeon et al., 2008) by going beyond discussions of symptom clusters to the underlying causes of the symptoms. In whatever fashion complaints and symptoms are grouped, attributing them to causes, wherever possible, is of critical importance. Evidently, understanding and treating DP/DR-D, as with other mental disorders, necessitates knowing its causes, contents, and consequences, including the causal links between these causes and the observed "symptoms." Clinically, the circumplex model indicates that a "one size fits all" approach to treatment is invalid and highlights developing individual formulations of DP/DR-D patients, based on an understanding of the specific mechanisms of the problem in question. Other forms of treatment, for example, may be more appropriate for addressing DP/DR associated with altered perceived causality (see Targ et al., 2000).

In sum, considering depersonalization/derealization as multidimensional necessitates a comprehensive examination of the intricate causal relationships between DP/DR and affective, cognitive, and behavioral constructs. Several of the results of the review highlight the importance of specifying causes of DP/DR that moderate its relationship with negative affect. Given these findings, it is imperative that researchers take a multidimensional contextualized approach to the study of DP/DR so our theoretical understanding of these phenomena can be refined.

\subsection{Normal and Abnormal Depersonalization/Derealization}

Second (and more important relative to the discussion), the taxonomy presented here clarifies thinking about normal and pathological DP/DR. According to Steinberg (1995) although "normal" and "pathological" DP share some aspects (i.e., feelings of unreality, detachment, strangeness), the quality of pathological DP has a key distinguishing feature. Steinberg has described the distinguishing feature of pathological DP as dissociation between an "observing" and "experiencing" self. Normal DP/DR, however, appears to be a transient alteration of consciousness with no dissociation between an observing and experiencing self. The experiencing self consists of body, thoughts, feelings, memories, and emotions. The observing self is experienced as a separate, uninvolved "critic" of the experiencing self, with the perception that all the normal aspects of personality are unreal and do not belong to the observing self. Instead of a synthesis of the observing and experiencing self, the observing self is detached from what the experiencing or participating self is doing. Patients describe their detached observation of experience as if they were "watching" some other self behave. Steinberg's research suggests it is both the persistence and the nature of DP/DR that differ from normal to abnormal subjects. Other authors have also proposed to distinguish dissociation between an observing and experiencing self from other symptoms of DP/DR (Van der Hart, Nijenhuis, Steele, \& Brown, 2004).

The data in Figure 1 suggest the only cause capable of explaining both the persistence and nature of pathological depersonalization is heightened self-focus. Self-focus involves an act of reflexivity or the capacity to take into account one's own consciousness, that is, the capacity to take the self as object. As William James long ago pointed out in his seminal discussion of the self (1890), each time we reflect upon ourselves as an object we 
perform an act of dissociation between the self as object (or observing self) and self as subject (or experiencing self). While reflexivity involves a focus on the self, it is the extent of self-focus that depends on whether reflexivity turns into a debilitating act. Unfortunately, self-focused attention may not always have an adaptive character. It would be naïve to propose that all DP/DR results from self-focus or any other single psychological process. Self-focus is proposed as only one form among several causal processes, but is suggested to be distinctive of pathological DP/DR. Table 1 contains the proposed assignment of DP/DR causes classified by degree of severity and is a modified version of that described by Steinberg (1995, p. 135). As depicted in Table 1, heightened self-focus is identified as the distinctive cause of pathological DP/DR. The table shows that most DP/DR can be classified as "common-mild." In some cases, transient symptoms may instigate the course of induction and maintenance of excessive self-focus.

DP/DR-D seems to be best described as a disorder of detachment involving an exaggerated and often unpleasant hyperawareness of the self (Simeon \& Abugel, 2006). Since Duval and Wicklund (1972) showed that self-focused attention promotes self-evaluation, a considerable amount of research has connected self-focus to aversive, dysfunctional, and problematic outcomes (Ingram, 1990; Wells \& Matthews, 1994). Ingram (1990) argued that self-focus plays a broad role in psychopathology. His review shows that a wide variety of disorders are instigated and maintained by chronic self-focus. The relationship of self-focus with general psychopathology and DP/DR could explain the high prevalence (between 40-80\%) of DP/DR in psychiatric patients (Hunter et al., 2004).

\subsection{Needed Areas of Research and Theory}

The circumplex-derived dimensions help identify areas of theoretical and empirical deficiency. Most notable are questions stemming from the relationship between dissociation and affect. Dissociation is believed to serve a protective function by allowing people to avoid experiencing the emotional impact of extreme threat. In their paper "Depersonalization Disorder: Dissociation and Affect," however, Simeon, Riggio-Rosen, Guralnik, Kniitelska, and Nelson (2003) describe study results suggesting that dissociation does not serve a protective function. Compared to control participants, patients with DP/DR-D actually suffered from chronic elevations in frequency and intensity of negative affect. Alternatively, self-focus may explain the correlation between DP/DR-D and chronic elevations of negative affect. Patients with DP/DR-D can be characterized by a tendency to strongly introspect and reflect on their self, that is, heightened self-focus. Clinically, this increased self-focus has been implicated as a major cause of negative affect (Ingram, 1990).

Despite the well-known role self-focus plays in DP/DR-D, the underlying mechanisms remain unclear. Thus, a priority for future research should be the systematic examination of the self-focused attention process within DP/DR-D. Toward this end, much has been written about the necessity of understanding self-focused attention as part of an overall self-regulatory process (Carver \& Scheier, 1998; Pyszczynski, Greenberg, Hamilton, \& Nix, 1991). Carver and Scheier (1998) suggested that self-focus plays a self-regulatory role that helps people in the pursuit of goals. In this self-regulatory process, people compare their current standing with self-standards and determine whether they are meeting this standard. If the current self matches the desired standard, the person terminates the regulatory process. If, however, progress toward the standard is slow or the discrepancy between the current self and the desired standard seems impossible to bridge, negative affect results. One context in which this discrepancy is particularly salient is stressful life events. Carver and Scheier (1998) also discussed the bidirectionality of the relationship between self-focused attention and negative affect and the role negative affect plays in drawing attention to the self.

Another topic needing more extended discussion is the relationship between DP/DR and altered perceived causality. It has been suggested that a breakdown in the normal ability to filter out irrelevant sensory stimuli from the environment may contribute to cognitive fragmentation in psychotic disorders (McGhie \& Chapman, 1961). These "sensorimotor gating" deficits can lead to sensory flooding and an array of psychotic symptoms as indicated by ideas of reference, odd beliefs, and magical thinking. DP/DR during the prodromal and acute phase of psychosis is possibly related to the similar, if not identical, character of magical thinking and unreality experiences, and this topic certainly deserves further exploration.

Finally, future research should attempt to establish (causal) relationships among the more or less standard set of DP/DR precipitators. In other words, how do the different DP/DR precipitants interact? Various combinations of precipitators may synergistically interact, with one increasing the potential intensity of the other. In essence, the interacting variables need to be modeled for clearer theoretical interpretation. This points to the need for new treatment models that specifically address the processes engaged in DP/DR phenomena. 


\section{Conclusions}

The literature reveals that DP/DR is associated with a wide range of altered states of consciousness. The cognitive conception of DP/DR describes these experiences as unidimensional with cognitive misinterpretations perpetuating normally transient DP/DR. The available empirical and conceptual evidence reviewed in this paper is more consistent with a multidimensional view of DP/DR characterized by the dimensions of valence and arousal. This review supports the contention that abnormal DP/DR has a distinguishing qualitative feature, namely, dissociation between an observing and experiencing self. Further, it appears that heightened self-focus uniquely precipitates and maintains this type of dissociation. It is hoped that this review will contribute not only to a better understanding of DP and DR but also to the continued development of the understanding of normal and abnormal functioning.

\section{References}

Ackner, B. (1954). Depersonalization, I: Aetiology and phenomenology. Journal of Mental Science, 100, 838-853. doi:10.1192/bjp.100.421.838

Aderibigbe, Y. A., Bloch, R. M., \& Walker, W. R. (2001). Prevalence of depersonalization and derealization experiences in a rural population. Social Psychiatry and Psychiatric Epidemiology, 36(2), 63-69. doi:10.1007/s001270050291

American Psychiatric Association. (1994). Diagnostic and statistical manual of mental disorders (4th ed.). Washington, DC: American Psychiatric Association.

Balaban, C. D., \& Jacob, R. G. (2001). Background and history of the interface between anxiety and vertigo. Journal of Anxiety Disorders, 15(1-2), 27-51. doi:10.1016/S0887-6185(00)00041-4

Barlow, D. H. (1988). Anxiety and its disorders. New York: Guilford Press.

Baumeister, R. F. (1991). Escaping the self. New York: Basic Books.

Becker-Blease, K. A. (2004). Dissociative states through new age and electronic trance music. Journal of Trauma and Dissociation, 5(2), 89-100. doi:10.1300/J229v05n02_05.

Beere, D. B. (1995a). Loss of "Background": A perceptual theory of dissociation. Dissociation, 8, 166-174.

Beere, D. B. (1995b). Dissociative reactions and characteristics of the trauma: Preliminary tests of a perceptual theory of dissociation. Dissociation, 8, 175-202.

Bliss, E., Clark, L., \& West, C. (1959). Studies of sleep deprivation: Relationship to schizophrenia. Archives of Neurologica Psychiatry, 81, 348-359.

Brown, A. S. (2004). The déjà vu experience. Hove, UK: Psychology Press.

Cameron, C. (1973). A theory of fatigue. Ergonomics, 16(5), 633-648.

Carver, C. S. (2001). Affect and the functional bases of behavior: On the dimensional structure of affective experience. Personality and Social Psychology Review, 5, 345-356.

Carver, C. S., \& Scheier, M. F. (1998). On the self-regulation of behavior. New York: Cambridge University Press.

Castillo, R. J. (1990). Depersonalization and meditation. Psychiatry, 53(2), 158-168.

Devinsky, O., Putnam, F., Grafman, J., Bromfield, E., \& Theodore, W. H. (1989). Dissociative states and epilepsy. Neurology, 39(6), 835-40.

Duval, S., \& Wicklund, R. A. (1972). A theory of objective self-awareness. New York: Academic Press.

Fewtrell, W. D., \& O’Connor, K. P. (1988). Dizziness and depersonalization. Advances in Behaviour Research and Therapy, 10, 201-218. doi:10.1016/0146-6402(88)90015-X

Fischer, R. (1971). A cartography of ecstatic and mystical states. Science, 174, 897-904. doi:10.1126/science.174.4012.897

Giesbrecht T., \& Merckelbach, H. (2004). Subjective sleep experiences are related to dissociation. Personality and Individual Differences, 37, 1341-1345. doi:10.1016/j.paid.2004.01.004

Giesbrecht, T., Lynn, S. J., Lilienfeld, S. O., \& Merckelbach, H. (2010). Cognitive Processes, Trauma, and Dissociation-Misconceptions and Misrepresentations: Reply to Bremner. Psychological Bulletin, 134(1), 7-11. doi:10.1037/a0018068 
Grigsby, J. P., \& Johnston, C. L. (1989). Depersonalization, vertigo and Ménière's disease. Psychological Reports, 64(2), 527-34.

Grigsby, J., \& Kaye, K. (1993). Incidence and correlates of depersonalization following head trauma. Brain Injury, 7, 507-513. doi:10.3109/02699059309008178

Gruzelier, J. H., Doig, A. (1996). The factorial structure of schizotypy: Part II. Cognitive asymmetry, arousal, handedness, and sex. Schizophrenia Bulletin, 22(4), 621-634. doi:10.1093/schbul/22.4.621

Harper, M., \& Roth, M. (1962). Temporal lobe epilepsy and the phobic anxiety-depersonalization syndrome. Comprehensive Psychiatry, 3(3), 129-151. doi:10.1016/S0010-440X(62)80009-1

Harrison, Y., \& Horne, J. A. (2000). Sleep loss and temporal memory. Quarterly Journal of Experimental Psychology, 53(1), 271-279. doi:10.1080/027249800390772

Hoffer, M. E., Balough, B. J., \& Gottshall, K. R. (2007). Posttraumatic balance disorders. International Tinnitus Journal, 13(1), 69-72.

Hood, R. W., Jr. (1976). Conceptual criticisms of regressive explanations of mysticism. Review of Religious Research, 17(3), 179-188.

Hull, J. G. (1987). Self-awareness model. In H. T. Blane \& K. E. Leonard (Eds.), Psychological theories of drinking and alcoholism (pp. 272-30). New York: Guilford Press.

Hunter, E. C., Phillips, M. L., Chalder, T., Sierra, M., \& David, A. S. (2003). Depersonalisation disorder: A cognitive-behavioural conceptualisation. Behaviour Research and Therapy, 41(12), 1451-1467. doi:10.1016/S0005-7967(03)00066-4

Hunter, E. C., Sierra, N. I., \& David, A. S. (2004). The epidemiology of depersonalisation and derealisation: A systematic review. Social Psychiatry and Psychiatric Epidemiology, 39(1), 9-18. doi:10.1007/s00127-004-0701-4

Ingram, R. E. (1990). Self-focused attention in clinical disorders: Review and a conceptual model. Psychological Bulletin, 107(2), 156-176. doi:10.1037/0033-2909.107.2.156

James, W. (1890). The principles of psychology. New York: Holt.

Kenna, J. C., \& Sedman, G. (1965). Depersonalisation in temporal lobe epilepsy and the organic psychoses. British Journal of Psychiatry, 111, 293-299. doi:10.1192/bjp.111.473.293

Kennedy, J. E., \& Kanthamani, H. (1995). An exploratory study of the effects of paranormal and spiritual experience on peoples' lives and well-being. Journal of the American Society for Psychical Research, 89(3), 249-264.

Lambert, M. V., Senior, C., Phillips, M. L., \& David, A. S. (2000). Depersonalization in Cyberspace. The Journal of Nervous and Mental Disease, 188(11), 764-771.

Lazarus, R. (1974). The psychology of coping: Issues of research and assessment. In G. V. Coelho, D. A. Hamburg, \& J. E. Adams (Eds.), Coping and adaptation (pp. 47-68). New York: Basic Books.

Lickel, J., Nelson, E., Lickel, A. H., \& Deacon, B. (2008). Interoceptive exposure exercises for evoking depersonalization and derealization: A Pilot Study. Journal of Cognitive Psychotherapy: An International Quarterly, 22(4), 321-330. doi:10.1891/0889-8391.22.4.321

Maslach, C. (2003). Burnout: The cost of caring. Cambridge: Malor Books.

Maslach, C., \& Jackson, S. E. (1986). Maslach burnout inventory (2nd ed.). Palo Alto, CA: Consulting Psychologists Press.

Mathew, R. J., Wilson, W. H., Chiu, N. Y., Turkington, T. G., Degrado, T. R., \& Coleman, R. E. (1999). Regional cerebral blood flow and depersonalization after tetrahydrocannabinol administration. Acta Psychiatrica Scandinavica, 100(1), 67-67. doi:10.1111/j.1600-0447.1999.tb10916.x

McGhie, A., \& Chapman, J. (1961). Disorders of attention and perception in early schizophrenia. British Journal of Medical Psychology, 34, 103-116.

Medford, N., Baker, D., Hunter, E., Sierra, M., Lawrence, E., Phillips, M. L., \& David, A. S. (2003). Chronic depersonalization following illicit drug use: A controlled analysis of 40 cases. Addiction, 98(12), 1731-1736. doi:10.1111/j.1360-0443.2003.00548.x 
Melges, F. T., Tinklenberg, J. R., Deardorff, C. M., Davies, N. H., Anderson, R. E., \& Owen, C. A. (1974). Temporal disorganization and delusional-like ideation. Processes induced by hashish and alcohol. Archives of General Psychiatry, 30(6), 855-861.

Melges, F. T., Tinklenberg, J. R., Hollister, L. E., \& Gillespie, H. K. (1970a). Marihuana and temporal disintegration. Science, 168(935), 1118-1120. doi:10.1126/science.168.3935.1118

Melges, F. T., Tinklenberg, J. R., Hollister, L. E., \& Gillespie, H. K. (1970b). Temporal disintegration and depersonalization during marijuana intoxication. Archives of General Psychiatry, 23(3), 204-210.

Neuhauser, H. K., \& Lempert, T. (2009). Vertigo: Epidemiologic aspects. Seminars in Neurology, 29(5), 473-81. doi:10.1055/s-0029-1241043

Noyes, R., \& Kletti, R. (1977). Depersonalization in response to life-threatening danger. Comprehensive Psychiatry, 18(4), 375-384. doi:10.1016/0010-440X(77)90010-4

Oswald, I. (1970). Sleep. London: Penguin Books.

Parnas, J., \& Sass, L. A. (2001). Self, solipsism, and schizophrenic delusions. Philosophy, Psychiatry, and Psychology, 8(2), 101-120. doi:10.1353/ppp.2001.0014

Penfield, W., \& Rasmussen, T. (1950). The cerebral cortex of man: A clinical study of localization of function. New York: Macmillan.

Pica, M., \& Beere, D. B. (1995). Dissociation during positive situations. Dissociation, 8, 241-246.

Pyszczynski, T., Greenberg, J., Hamilton, J., \& Nix, G. (1991). On the relationship between self-focused attention and psychological disorder: A critical reappraisal. Psychological Bulletin, 110(3), 538-543. doi:10.1037/0033-2909.110.3.538

Ravizza, K. (1977). Peak experience in sport. Journal of Humanistic Psychology, 17(4), 35-40. doi: $10.1177 / 002216787701700404$

Reed, G., \& Sedman, G. (1964). Personality and depersonalization under sensory deprivation conditions. Perceptual and Motor Skills, 18, 659-660.

Russell, J. A. (1980). A circumplex model of affect. Journal of Personality and Social Psychology, 39(6), 1161-1178. doi:10.1037/h0077714

Russell, J. A., \& Barrett, L. F. (1999). Core affect, prototypical emotional episodes, and other things called emotion: Dissecting the elephant. Journal of Personality and Social Psychology, 76(5), 805-819. doi:10.1037/0022-3514.76.5.805

Sass, L. A. (1994). The paradoxes of delusion: Wittgenstein, Schreber, and the schizophrenic mind. Ithaca, NY: Cornell University Press.

Sierra, M. (2009). Depersonalization: A new look at a neglected syndrome. New York: Cambridge University Press.

Sierra, M., Baker, D., Medford, N., \& David, A. S. (2005). Unpacking the depersonalization syndrome: An exploratory factor analysis on the Cambridge Depersonalization Scale. Psychological Medicine, 35(10), 1523-1532. doi:10.1017/S0033291705005325

Sierra, M., \& Berrios, G. E. (1998). Depersonalization: Neurobiological perspectives. Biological Psychiatry, 44(9), 898-908. doi:10.1016/S0006-3223(98)00015-8

Sierra, M., Senior, C., Dalton, J., McDonough, M., Bond, A., Phillips, M. L., ... David, A. S. (2002). Autonomic response in depersonalization disorder. Archives of General Psychiatry, 59(9), 833-838. doi:10.1001/archpsyc.59.9.833

Simeon, D., \& Abugel, J. (2006). Feeling unreal: Depersonalization disorder and the loss of the self. New York: Oxford University Press.

Simeon, D. (2007). Relationships between dissociation and posttraumatic stress disorder. In E. Vermetten, M. J. Dorahy, \& D. Spiegel (Eds.), Traumatic dissociation: neurobiology and treatment (pp. 77-102). Washington, DC: American Psychiatric Publishing Group.

Simeon, D., \& Hamilton, H. K. (2008). Depersonalization disorder and schizotypal personality disorder. In A. Moskowitz, I. Schäfer, M. J. Dorahy (Eds.), Psychosis, trauma and dissociation: Emerging perspectives on severe psychopathology (pp. 209-220). West Sussex, UK: John Wiley and Sons. 
Simeon, D., Kozin, D. S., Segal, K., Lerch, B., Dujour, R., \& Giesbrecht, T. (2008). Deconstructing depersonalization: Further evidence for symptom clusters. Psychiatry Research, 157(1-3), 303-306. doi:10.1016/j.psychres.2007.07.007

Simeon, D., Riggio-Rosen, A., Guralnik, O., Kniitelska, M., \& Nelson, D. (2003). Depersonalization disorder: Dissociation and affect. Journal of Trauma and Dissociation, 4(4), 63-76. doi:10.1300/J229v04n04_05

Spiegel, H., \& Spiegel, D. (2004). Trance and treatment: Clinical uses of hypnosis (2nd ed.). Washington, DC: American Psychiatric Publishing.

Steinberg, M. (1995). Handbook for the assessment of dissociation: A clinical guide. Washington, DC: American Psychiatric Press.

Sterlini, G. L., \& Bryant, R. A. (2002). Hyperarousal and dissociation: A study of novice skydivers. Behaviour Research \& Therapy, 40(4), 431-437. doi:10.1016/S0005-7967(01)00021-3

Targ, E., Schlitz, M., \& Irwin, H. J. (2000). Psi-related experiences. In E. Cardeña, S. J. Lynn, \& S. Krippner (Eds.), Varieties of anomalous experience: Examining the scientific evidence (pp. 219-252). Washington, DC: American Psychological Association. doi:10.1037/10371-007

Torch, E. (1978). Review of the relationship between obsession and depersonalization. Acta Psychiatrica Scandinavica, 58(2), 191-198. doi:10.1111/j.1600-0447.1978.tb06931.x

Torch, E. (1981). Depersonalization syndrome: An overview. Psychiatric Quarterly, 53(3), 249-258. doi:10.1007/BF01070099

Trueman, D. (1984). Depersonalization in a non-clinical population. Journal of Psychology, 116, 107-112.

Vaitl, D., Birbaumer, N., Gruzelier, J., Jamieson, G. A., Kotchoubey, B., Kubler, A., ... Weiss, T. (2005). Psychobiology of altered states of consciousness. Psychological Bulletin, 131, 98-127. doi:10.1037/0033-2909.131.1.98

Van der Hart, O., Nijenhuis, E. R. S., Steele, K., \& Brown, D. (2004). Trauma-related dissociation: Conceptual clarity lost and found. Australian and New Zealand Journal of Psychiatry, 38(11-12), 906-914. doi:10.1111/j.1440-1614.2004.01480.x

Van IJzendoorn, M. H., \& Schuengel, C. (1996). The measurement of dissociation in normal and clinical populations: Meta-analytic validation of the Dissociative Experience Scale (DES). Clinical Psychology Review, 16, 365-382. doi:10.1016/0272-7358(96)00006-2

Vollenweider, F. X., Vontobel, P., Hell, D., \& Leenders, K. L. (1999). 5-HT modulation of dopamine release in basal ganglia in psilocybin-induced psychosis in man-A PET study with [11C]raclopride. Neuropsychopharmacology, 20, 424-433. doi:10.1016/S0893-133X(98)00108-0

Wallace, R. K. (1970). Physiological effects of transcendental meditation. Science, 167(926), 1751-1754. doi:10.1126/science.167.3926.1751

Watson, D. (2001). Dissociations of the night: Individual differences in sleep-related experiences and their relation to dissociation and schizotypy. Journal of Abnormal Psychology, 110(4), 526-35. doi:10.1037/0021-843X.110.4.526

Wells, A., \& Matthews, G. (1994). Attention and emotion: A clinical perspective. Hillsdale, NJ: Erlbaum.

Williams, D. (1956). The structure of emotions reflected in epileptic experiences. Brain, 79, 29-67. doi:10.1093/brain/79.1.29

Wineburg, E., \& Straker, N. (1973). An episode of acute, self-limiting depersonalization following a first session of hypnosis. American Journal of Psychiatry, 130, 98-100. doi:10.1176/appi.ajp.130.1.98

World Health Organization. (1992). The ICD-10 classification of mental and behavioral disorders: Clinical descriptions and diagnostic guidelines. Geneva, Switzerland: World Health Organization.

Yoo, S. S., Gujar, N., Hu, P., Jolesz, F. A., \& Walker, M. P. (2007). The human emotional brain without sleep: A prefrontal amygdala disconnect. Current Biology, 17(20), R877-R878. doi:10.1016/j.cub.2007.08.007

Zablocki, B., Aidala, A., Hansell, S., \& White, H. R. (1991). Marijuana use, introspectiveness, and mental health. Journal of Health and Social Behavior, 32(1), 65-89. 
Table 1. Comparison of Normal and Abnormal Depersonalization/Derealization

\begin{tabular}{|c|c|c|c|}
\hline Dimension & $\begin{array}{l}\text { Common-mild } \\
\text { depersonalization/ } \\
\text { derealization }\end{array}$ & $\begin{array}{l}\text { Transient } \\
\text { depersonalization/ } \\
\text { derealization }\end{array}$ & $\begin{array}{l}\text { Pathological } \\
\text { depersonalization/ } \\
\text { derealization }^{\mathrm{a}}\end{array}$ \\
\hline $\begin{array}{l}\text { Precipitating } \\
\text { factors }\end{array}$ & $\begin{array}{l}\text { - Sports } \\
\text { - Dancing } \\
\text { - Overwhelming joy } \\
\text { - Positive drug experiences } \\
\text { - Hypnotic induction } \\
\text { - Sensory deprivation } \\
\text { - Meditation } \\
\text { - Hypnagogic and } \\
\text { - Hypnopompic states } \\
\text { - Sligue } \\
\text { - Epilepsy } \\
\text { - Vertigo } \\
\text { - Hyperventilation }\end{array}$ & $\begin{array}{l}\text { - Stress } \\
\text { - Altered perceived causality } \\
\text { - Negative drug experiences } \\
\text { - Traumatic memory } \\
\text { - Extreme threat }\end{array}$ & - Heightened self-focus \\
\hline Context & Occurs as an isolated symptom & Occurs as an isolated symptom & $\begin{array}{l}\text { Occurs with depression, anxiety, } \\
\text { and dissociation }\end{array}$ \\
\hline Frequency & One-Few episodes & One-Few episodes & Recurrent-Persistent episodes \\
\hline Duration & $\begin{array}{l}\text { Brief episodes; lasts seconds to } \\
\text { minutes }\end{array}$ & $\begin{array}{l}\text { Limited duration (minutes to } \\
\text { weeks) }\end{array}$ & $\begin{array}{l}\text { Chronic lasting up to months or } \\
\text { years }\end{array}$ \\
\hline
\end{tabular}

a Pathological depersonalization/derealization is not expected to correlate with the precipitating factors listed in Column 1. It may, however, be precipitated by factors listed in Column 2 (e.g., stress, negative drug experiences) 


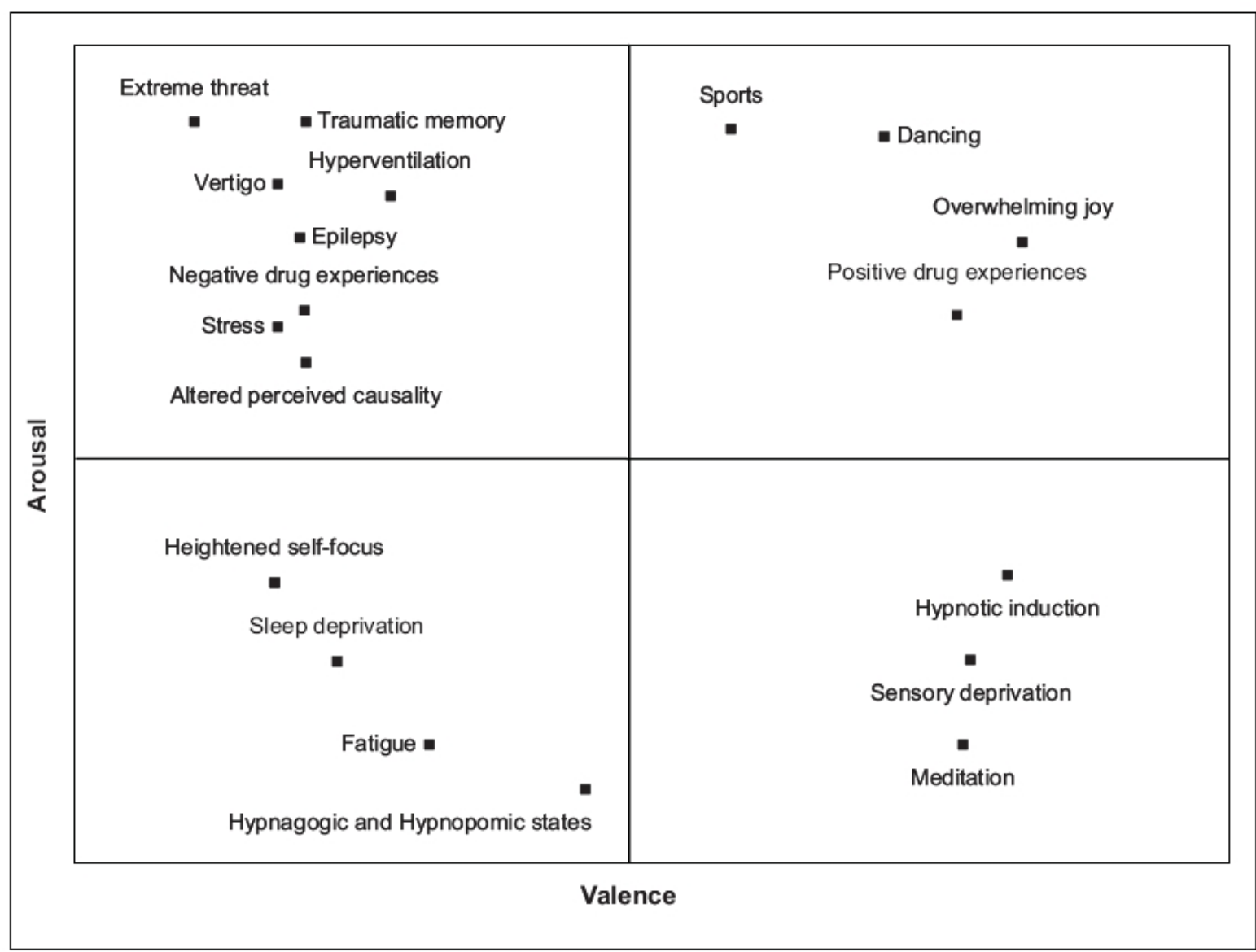

Figure 1. Graphic representation of circumplex structure of depersonalization/derealization.

Note: The horizontal axis indicates the valence (left $=$ negative valence, right $=$ positive valence), and vertical axis indicates the arousal level (top $=$ high arousal, bottom $=$ low arousal). 